\title{
QTL and genetic analysis controlling fiber quality traits using paternal backcross population in upland cotton
}

\author{
MA LingLing, SU Ying, NIE Hushuai, CUI Yupeng, CHENG Cheng, IJAZ Babar and HUA Jinping ${ }^{*}$ (D
}

\begin{abstract}
Background: Genetic improvement in fiber quality is one of the main challenges for cotton breeders. Quantitative trait loci (QTL) mapping provides a powerful approach to dissect the molecular mechanism in fiber quality traits. In present study, $F_{14}$ recombinant inbred line (RIL) population was backcrossed to paternal parent for a paternal backcross (BC/P) population, deriving from one upland cotton hybrid. Three repetitive BC/P field trials and one maternal backcross (BC/M) field trial were performed including both two BC populations and the original RIL population.
\end{abstract}

Results: In total, 24 novel QTLs are detected for fiber quality traits and among which 13 QTLs validated previous results. Thirty-five QTLs in BC/P populations explain 5.01\%-22.09\% of phenotype variation (PV). Among the 35 QTLs, 23 QTLs are detected in BC/P population alone. Present study provides novel alleles of male parent for fiber quality traits with positive genetic effects. Particularly, aFS-Chr3-1 explains $22.09 \%$ of PV in BC/P population, which increaseds $0.48 \mathrm{cN} \cdot$ tex $^{-1}$ for fiber strength. A total of 7, 2, 8, 2 and 6 QTLs explain over 10.00\% of PV for fiber length, fiber uniformity, fiber strength, fiber elongation and fiber micronaire, respectively. In RIL population, six common QTLs are detected in more than one environment: qFL-Chr1-2, qFS-Chr5-1, qFS-Chr9-1, qFS-Chr21-1, qFM-Chr9-1 and qFM-Chr9-2. Two common QTLs of qFE-Chr2-2 (TMB2386-SWU12343) and qFM-Chr9-1 (NAU2873-CGR6771) explain 22.42\% and 21.91\% of PV. The region between NAU4034 and TMB1296 harbor 30 genes (379 kb) in A05 and 42 genes (49 kb) in D05 for fiber length along the QTL GFL-Chr5-1 in BC/P population, respectively. In addition, a total of 142 and 46 epistatic QTLs and QTL $\times$ environments (E-QTLs and QQEs) are identified in recombinant inbred lines in paternal backcross (RIL-P) and paternal backcross (BC/P) populations, respectively.

Conclusions: The present studies provide informative basis for improving cotton fiber quality in different populations.

Keywords: Fiber quality traits, Common QTL, Paternal backcross population, Upland cotton

\section{Background}

Upland cotton (Gossypium hirsutum L.) is one of the most important sources of natural textile fiber. Among four cultivated Gossypium species, upland cotton shows higher yield potential and stronger adaptation to diverse

\footnotetext{
* Correspondence: jinping_hua@cau.edu.cn

Laboratory of Cotton Genetics, Genomics and Breeding /Beijing Key Laboratory of Crop Genetic Improvement /Key Laboratory of Crop Heterosis and Utilization of Ministry of Education, College of Agronomy and Biotechnology, China Agricultural University, Beijing 100193, China
}

environments than sea island cotton (G. barbadense), and accounts for more than $92 \%$ output of world cotton cultivation (Zhang et al. 2015a). However, fiber quality of upland cotton is not as good as that of sea island cotton. To meet the diverse demands of textile industry, improving fiber quality is a key target in upland cotton breeding projects.

Generally, main fiber quality traits consist of fiber length (FL), fiber uniformity (FU), fiber strength (FS), fiber elongation (FE), and fiber micronaire (FM). Each trait has its own genetic mechanism (Ijaz et al. 2019).

(c) The Author(s). 2020 Open Access This article is licensed under a Creative Commons Attribution 4.0 International License, which permits use, sharing, adaptation, distribution and reproduction in any medium or format, as long as you give appropriate credit to the original author(s) and the source, provide a link to the Creative Commons licence, and indicate if changes were made. The images or other third party material in this article are included in the article's Creative Commons licence, unless indicated otherwise in a credit line to the material. If material is not included in the article's Creative Commons licence and your intended use is not permitted by statutory regulation or exceeds the permitted use, you will need to obtain permission directly from the copyright holder. To view a copy of this licence, visit http://creativecommons.org/licenses/by/4.0/. 
Among 4892 QTLs in Cotton QTLdb database (Yu et al. 2014), 494, 289, 470, 287 and 395 were detected for FL, FU, FS, FE and FM, respectively (http://www2.cottonqtldb.org:8081/, Cotton QTLdb, the newly released version V2.3 on January 24, 2018).

A total of 151, 132, 91, 118 and 234 QTLs were metaanalyzed for QTL-rich regions for FL, FU, FS, FE and FM, respectively (Said et al. 2013). A number of QTLs were located on Chr 5, Chr 19 and Chr 21 (Said et al. 2013, 2015). For fiber quality, numerous QTLs were detected based on mapping in recombinant inbred lines (RIL) populations of upland cotton (Wu et al. 2009; Sun et al. 2012; Ning et al. 2014; Tan et al. 2015; Shang et al. 2015; Tang et al. 2015; Zhang et al. 2015b; Jamshed et al. 2016; Li et al. 2016; Ma et al. 2017; Ijaz et al. 2019). However, RIL population can only be dissected additive and additive $x$ additive effects, not to be dissected dominance and dominance-related genetic effects due to lacking heterozygous genotypes. Recently, the permanent RIL populations were used to develop backcross populations in rice (Mei et al. 2005) and in cotton (Shang et al. 2016d). The backcross populations allow performing repetitive trials as doing in 'immortalized' $F_{2}$ population (Hua et al. 2002, 2003). Seven QTLs were detected for fiber length and fiber strength by using backcross population deriving from Guazuncho $2 \times$ VH8-4602 (Lacape et al. 2005). A total of 44 QTLs were detected for fiber quality traits on Chr 1 , Chr 9 and $\mathrm{Chr}$ 21 using (CCRI $8 \times$ Pima 90-53) $\times$ CCRI $8 \quad B_{1} F_{1}$ interspecific population (Yang et al. 2015). Shang et al. (2016d) detected 17, 6, 15, 11 and 21 QTLs for FL, FU, $\mathrm{FS}, \mathrm{FE}$ and $\mathrm{FM}$, respectively, in $\mathrm{F}_{9}$ RIL and $\mathrm{F}_{9} \mathrm{BC}_{1}$ progenies of a hybrid 'Xinza 1'. Wang et al. (2016) detected $22,14,17,3$ and 20 QTLs for the five traits in two parental $\mathrm{F}_{8} \mathrm{BC}_{1}$ populations deriving from another hybrid of upland cotton. In previous study, two markers of NAU5530 and CIR099 flanking QTLs $q F L-c 19-2$, $q F U$ LG3-1 and qFS-LG3 were the same as those in Shang's work (2016d). Using a map of single nucleotide polymorphism (SNP) markers, one fiber length hotspot was observed on Chr 5 carrying three QTLs (Li et al. 2016). Nineteen clusters harbored favorable alleles from G. barbadense for two or three fiber quality traits (Shi et al. 2020). Additionally, four potential candidate genes for fiber length on Chr Dt7 were found using genotyping by sequencing by genome-wide association studies (GWAS) (Su et al. 2016). Previous studies indicated that one RIL population and its two $\mathrm{BCF}_{1}$ populations could increase the power of QTL detection (Shang et al. 2016d; Wang et al. 2016). It offers opportunity to dissect QTLs for fiber quality traits using multiple populations of upland cotton.

Cotton genomes for diploid species (Paterson et al. 2012; Wang et al. 2012a; Li et al. 2014; Du et al. 2018) and tetraploid genomes (Li et al. 2015; Liu et al. 2015; Yuan et al. 2015; Zhang et al. 2015a) have been released recently. New versions of reference genomes have recently been released in cotton (Fang et al. 2017a; Wang et al. 2019; Hu et al. 2019). These genomic analyses in cotton facilitate applications of SNP markers (Ali et al. 2018) and GWAS for fiber quality (Fang et al. 2017b; $\mathrm{Ma}$ et al. 2018b). It is very important to detect novel QTLs and to validate the reported QTLs using diverse populations or accessions. In previous studies, serial genetic analyses were performed in multiple segregating populations across multiple years and various locations, including $\mathrm{F}_{2}, \mathrm{~F}_{2:}$, $\mathrm{RIL}$ and $\mathrm{BC} / \mathrm{M}$ population deriving from the hybrid 'Xinza 1' (Liang et al. 2013; Liang et al. 2015; Shang et al. 2015, 2016a, 2016b, 2016c, 2016d; Ma et al. 2017, 2018a, 2019). A total of 111 quantitative trait loci (QTLs) were detected for fiber quality traits using four populations derived from RIL (XZ) and backcross (XZV) hybrids (Shang et al. 2016d). A total of 55 QTLs were detected and found distributed in 21 chromosomes using $\mathrm{BC} / \mathrm{M}$ population in three locations ( $\mathrm{Ma}$ et al. 2017). In addition, 32 QTLs at five stages, 24 conditional QTLs at four intervals were both detected for plant height at different stages (Ma et al. 2018a). Meanwhile, 26 and 27 QTLs including heterotic loci were identified in $\mathrm{BC} / \mathrm{P}$ and $\mathrm{BC} / \mathrm{M}$ populations, respectively (Ma et al. 2019). In addition, 10 and 16 clusters were improved more than one trait for fiber quality and yieldcomponents, respectively ( $\mathrm{Ma}$ et al. 2017; Ma et al. 2019). In order to dissect genetic components of fiber quality, we developed $\mathrm{BCF}_{1}$ progenies population based on RIL population by backcrossing with the paternal parent of 'Xinza 1'. Here we term as paternal backcross population (BC/P population for short). We generated additional $177 \mathrm{BCF}_{1}$ crosses for $\mathrm{BC} / \mathrm{P}$ populations by backcrossing the 177 RI lines as current female parents to GX100-2 (the original male parent), respectively. Detection of novel QTLs and comparison analysis were performed for fiber quality traits using $\mathrm{BC} / \mathrm{P}, \mathrm{BC} / \mathrm{M}$ and RIL populations.

\section{Materials and methods}

\section{Plant materials and populations development}

The intraspecific $\mathrm{F}_{14}$ recombinant inbred lines (RIL) were inbred for 177 individuals by single seed descent method, which were derived from an upland cotton hybrid "Xinza 1" (GX 1135× GX 100-2) (Shang et al. 2016a). The parental backcross (BC/P) population was obtained by backcrossing the original male parent (GX100-2) to 177 RIL, respectively. The maternal backcross $(\mathrm{BC} / \mathrm{M})$ population is referred to previous studies (Ma et al. 2017; Ma et al. 2019). The GX100-2, "Xinza 1", GX1135 and a competition hybrid "Ruiza 816" in the Yellow River Region were performed in every 
experimental trial as the control set. In present study, we name the maternal and paternal backcross populations as $\mathrm{BC} / \mathrm{M}$ and $\mathrm{BC} / \mathrm{P}$ populations, respectively, so as RIL-M and RIL-P populations for the RIL population in four $\mathrm{BC} / \mathrm{M}$ and $\mathrm{BC} / \mathrm{P}$ field trials.

\section{Field trial arrangement, sampling and trait evaluation}

Four trials were performed at two locations in Hebei province, China: E1. Quzhou Experimental Station in Handan city; E2, Guoxin Seed Company Ltd. in Cangzhou city. Three BC/P field trials were conducted in 2015E2 (year + location), 2016E1, 2016E2. At the same time, one maternal BC trial was conducted in 2016E2 as control trial. The field trials were designed and planted identical to previous studes for $\mathrm{BC} / \mathrm{M}$ field trials in 2012E1, 2012E2 and 2012E4 (E4, Xiangyang city in Hubei province, China; Shang et al. 2016a), same as 2015E1, 2015E2, and 2015E3 (E3: Wuhan city in Hubei province, China; Ma et al. 2017, 2018a, 2019). Field management followed local conventional standard field practice.

Twenty-five naturally opening bolls in the middle of plants were hand-harvested for each plot at mature stage in all three environments. Fiber samples were ginned and sampled for measurements of fiber quality traits with HVI 900 instrument (Uster_HVISpectrum, Spinlab, USA) at Cotton Fiber Quality Inspection and Testing Center of Ministry of Agriculture and Rural Affairs (Anyang, China) (Shang et al. 2016d; Shahzad et al. 2019). Five fiber quality traits were measured, containing 2.5\% fiber span length ( $\mathrm{FL}, \mathrm{mm})$, fiber uniformity (FU, \%), fiber strength (FS, cN.tex ${ }^{-1}$ ), fiber elongation (FE), and fiber micronaire (FM) as usual (Zhang et al. 2005).

\section{Genetic map and data analysis}

Genetic map is based on RIL population published before (Shang et al. 2016c), in which a total of 653 loci based on SSR markers distributed on 31 linkage groups and anchored on 26 chromosomes, covering $3889.9 \mathrm{cM}$ $(88.20 \%)$ of cotton genome with average interval of 6.2 cM (Ma et al. 2017, 2018a, 2019). The genotype for each maternal $\mathrm{F}_{14} \mathrm{BC}_{1}$ was deduced on the basis of the RIL genotype (Shang et al. 2016a, 2016b, 2016c).

Basic statistical analysis was implemented by the software SPSS (Version 19.0, SPSS, Chicago). Using variance analysis, heritability was calculated according to the equation as $h^{2}=\delta_{G}^{2} /\left[\delta_{G}^{2}+\left(\delta_{G}^{2}{ }_{G}{ }_{E}\right) / e n v\right]$, where $\delta_{G}^{2}, \delta_{G \times E}^{2}$ and env refer to the genotypic variance, genotype-byenvironment interaction variance and the number of the environments, respectively.

Composite interval mapping (CIM) method was used for QTL mapping in the confidence interval of $95 \%$. The software QTL Cartographer (Version 2.5) (Zeng 1994; Wang et al. 2012b) was used to map single-locus QTL and to estimate the genetic effect. The threshold of logarithm of odds (LOD) was estimated to declare a suggestive QTL after 1000 permutation times, whereas QTL in another environment or population with LOD of at least 2.0 was considered as common QTL (Liang et al. 2013; Shang et al. 2015, 2016d). According to the position linking and sharing of common markers, QTLs detected in different populations were regarded as one common QTL (Shao et al. 2014; Shang et al. 2016d; Ma et al. 2017).

The QTL IciMapping 4.1 (www.isbreeding.net) was conducted by two-locus analysis using inclusive composite interval mapping (ICIM) method (Shang et al. 2016d; Ma et al. 2017). The main-effect QTL (M-QTL) and its environmental interaction (QTL $\times$ environment, $\mathrm{QE}$ ), epistatic QTLs (E-QTLs) and its environmental interactions (QTLs $\times$ environment, QQE) were conducted using RIL-P and $\mathrm{BC} / \mathrm{P}$ datasets under multiple environments in three $\mathrm{BC} / \mathrm{P}$ trials. A threshold LOD 2.5 and 5 scores were used to declare significant M-QTL and EQTLs, respectively.

\section{Results}

\section{Trait performance in two populations}

The phenotype of five fiber quality traits performed differently between the 'original' maternal parent 'GX1135' of Xinza 1 and the 'original' male parent 'GX100-2' (Table 1). The hybrid 'Xinza 1'showed no significant hybrid vigor of $F_{1}$ for fiber quality traits ranging from $3.17 \%$ to $1.53 \%$ of mid-parent heterosis (MPH). Phenotypic variation ranged from $2.63 \%$ to $8.53 \%$ in both BC and RIL populations for fiber length (FL), fiber strength (FS) and fiber micronaire (FM). However, the values ranged from $0.87 \%$ to $1.23 \%$ for fiber uniformity (FU) and fiber elongation (FE).

Genotype variance and environment variance showed significant variation for five traits at level of 0.05 in RIL and $\mathrm{BC}$ populations (Table 2). Fiber length (FL) and fiber uniformity $(\mathrm{FU})$ increased in $\mathrm{BC} / \mathrm{P}$ population in comparison with that in $\mathrm{BC} / \mathrm{M}$ population, whereas fiber micronaire (FM) reduced. Fiber length (FL) and fiber strength (FS) showed larger heritability of $91.82 \%$ and $91.10 \%$, respectively, in RIL-P population. The heritability decreased to $86.63 \%$ and $81.93 \%$ for FL and FS, respectively, in $\mathrm{BC} / \mathrm{P}$ population (Table 2). The results indicated wider range of phenotypic variation and larger heritability in RIL-P population than those in BC/P population for five fiber quality traits.

\section{Correlation analysis among fiber quality traits in multiple populations}

The significant correlation coefficients were calculated for five fiber quality traits in $\mathrm{BC} / \mathrm{P}, \mathrm{BC} / \mathrm{M}, \mathrm{RIL}-\mathrm{P}$ and RIL-M populations in 2015E2, 2016E1 and 2016E2 (Table 3). Fiber length (FL) correlated significantly and positively 


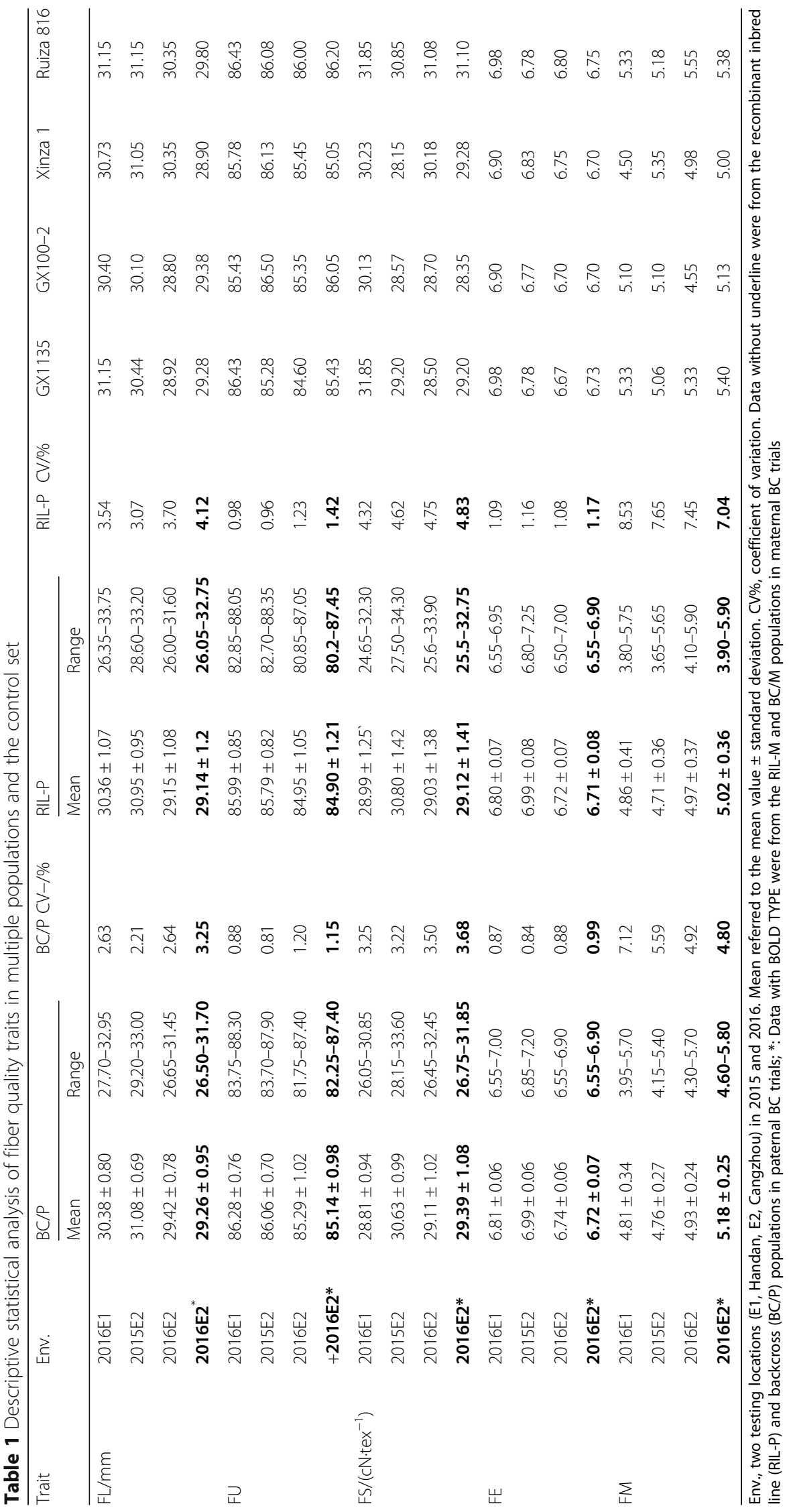


Table 2 Results of ANOVA and heritability for yield and its components in different populations from two backcross trials

\begin{tabular}{|c|c|c|c|c|c|c|c|c|c|c|}
\hline \multirow[t]{2}{*}{ Trait } & \multirow{2}{*}{$\begin{array}{l}\text { Source } \\
\text { of } \\
\text { variation }\end{array}$} & \multicolumn{5}{|l|}{ MS } & \multicolumn{4}{|l|}{ MS } \\
\hline & & $\overline{M S^{a}}$ RIL-P & $H^{2} / \%^{b}$ & $\mathrm{MS}^{\mathrm{a}} \mathrm{BC} / \mathrm{P}$ & $H^{2} / \% b$ & $\mathrm{BC} / \mathrm{P}$ & $\mathrm{MS}^{\mathrm{a}}$ RIL-M & $H^{2} / \%$ & $\mathrm{MS}^{\mathrm{a}} \mathrm{BC} / \mathrm{M}$ & $H^{2} / \%$ \\
\hline \multirow[t]{4}{*}{$\overline{\mathrm{FL}}$} & $G$ & $4.753^{* *}$ & 91.82 & $2.072^{* *}$ & 86.63 & $2.072^{* *}$ & $5.213^{* *}$ & 91.78 & $2.021^{* *}$ & 79.62 \\
\hline & E & $291.886^{* *}$ & & $240.054^{* *}$ & & $240.054^{* *}$ & $269.064^{* *}$ & & $297.752^{* *}$ & \\
\hline & $G \times E$ & 0.864 & & 0.652 & & 0.652 & 0.955 & & 1.053 & \\
\hline & Error & 0.814 & & 0.615 & & 0.615 & 0.888 & & 0.998 & \\
\hline \multirow[t]{4}{*}{ FU } & G & $1.880^{*}$ & 71.06 & $1.490^{*}$ & 69.35 & $1.490^{*}$ & $2.828^{* *}$ & 78.69 & $1.785^{*}$ & 72.00 \\
\hline & E & $110.536^{* *}$ & & $95.792^{* *}$ & & $95.792^{* *}$ & $259.923^{* *}$ & & $272.103^{* *}$ & \\
\hline & $G \times E$ & 1.55 & & 1.368 & & 1.368 & 1.524 & & 1.364 & \\
\hline & Error & 1.494 & & 1.214 & & 1.214 & 1.548 & & 1.437 & \\
\hline \multirow[t]{4}{*}{ FS } & G & $7.906^{* *}$ & 91.10 & $3.044^{* *}$ & 81.93 & $3.044^{* *}$ & $8.901^{* *}$ & 89.71 & $4.039^{* *}$ & 81.95 \\
\hline & E & $369.073^{* *}$ & & $333.408^{* *}$ & & $333.408^{* *}$ & $159.82^{* *}$ & & $85.482^{* *}$ & \\
\hline & $G \times E$ & $1.625^{*}$ & & 1.365 & & 1.365 & 2.07 & & 1.71 & \\
\hline & Error & 1.381 & & 1.298 & & 1.298 & 1.989 & & 1.919 & \\
\hline \multirow[t]{4}{*}{$\mathrm{FE}$} & G & $0.022^{* *}$ & 86.83 & $0.010^{* *}$ & 79.87 & $0.010^{* *}$ & $0.032^{* *}$ & 88.26 & $0.014^{* *}$ & 78.81 \\
\hline & E & $6.779^{* *}$ & & $6.063^{* *}$ & & $6.063^{* *}$ & $6.453^{* *}$ & & $6.614^{* *}$ & \\
\hline & $\mathrm{G} \times \mathrm{E}$ & 0.007 & & 0.005 & & 0.005 & $0.009^{* *}$ & & 0.008 & \\
\hline & Error & 0.006 & & 0.005 & & 0.005 & 0.007 & & 0.007 & \\
\hline \multirow[t]{4}{*}{ FM } & G & $0.576^{* *}$ & 89.00 & $0.261^{* *}$ & 81.25 & $0.261^{* *}$ & $0.439^{* *}$ & 85.33 & $0.202^{* *}$ & 76.37 \\
\hline & $E$ & $6.24^{* *}$ & & $2.636^{* *}$ & & $2.636^{* *}$ & $11.665^{* *}$ & & $13.089^{* *}$ & \\
\hline & $\mathrm{G} \times \mathrm{E}$ & $0.154^{* *}$ & & 0.117 & & 0.117 & 0.158 & & 0.131 & \\
\hline & Error & 0.119 & & 0.128 & & 0.128 & 0.137 & & 0.112 & \\
\hline
\end{tabular}

G genotype, E environment, $\mathrm{G} \times \mathrm{E}$, genotypexenvironment. ${ }^{\mathbf{a}}$ Mean square. ${ }^{\mathbf{b}}$ Heritability. ${ }^{\prime * \prime}$ and ${ }^{\prime * * \prime}$ indicate that the correlation is significant at 0.05 and 0.01 probability levels, respectively

Table 3 Correlation analyses between five fiber quality traits in RIL population and its BC progenies

\begin{tabular}{|c|c|c|c|c|c|c|c|c|c|}
\hline \multirow[t]{2}{*}{ Trait } & \multirow[t]{2}{*}{ Env. } & \multicolumn{2}{|l|}{$\mathrm{FL}$} & \multicolumn{2}{|l|}{$\mathrm{FU}$} & \multicolumn{2}{|l|}{ FS } & \multicolumn{2}{|l|}{$\mathrm{FE}$} \\
\hline & & $\overline{\mathrm{RIL}}$ & $B C$ & $\overline{\mathrm{RIL}}$ & $B C$ & $\overline{\mathrm{RIL}}$ & $B C$ & $\overline{\mathrm{RIL}}$ & $B C$ \\
\hline \multirow[t]{4}{*}{$\overline{F U}$} & 2016E1 & 0.013 & -0.096 & & & & & & \\
\hline & 2015E2 & $0.226^{* *}$ & $0.222^{* *}$ & & & & & & \\
\hline & 2016E2 & $0.497^{* *}$ & $0.381^{* *}$ & & & & & & \\
\hline & $2016 \mathrm{E} 2^{*}$ & $0.438^{* *}$ & $0.178^{*}$ & & & & & & \\
\hline \multirow[t]{4}{*}{ FS } & 2016E1 & $0.656^{* *}$ & $0.616^{* *}$ & -0.030 & -0.083 & & & & \\
\hline & 2015E2 & $0.718^{* *}$ & $0.699^{* *}$ & $0.217^{* *}$ & 0.075 & & & & \\
\hline & 2016E2 & $0.754^{* *}$ & $0.581^{* *}$ & $0.403^{* *}$ & 0.060 & & & & \\
\hline & $2016 \mathrm{E} 2^{*}$ & $0.784^{* *}$ & $0.525^{* *}$ & $0.305^{* *}$ & 0.043 & & & & \\
\hline \multirow[t]{4}{*}{$\mathrm{FE}$} & 2016E1 & $0.659^{* *}$ & $0.617^{* *}$ & 0.128 & 0.105 & $0.530^{* *}$ & $0.511^{* *}$ & & \\
\hline & 2015E2 & $0.627^{* *}$ & $0.476^{* *}$ & $0.369^{* *}$ & $0.163^{*}$ & $0.507^{* *}$ & $0.533^{* *}$ & & \\
\hline & 2016E2 & $0.754^{* *}$ & $0.737^{* *}$ & $0.454^{* *}$ & $0.252^{* *}$ & $0.710^{* *}$ & $0.696^{* *}$ & & \\
\hline & $2016 \mathrm{E} 2^{*}$ & $0.813^{* *}$ & $0.535^{* *}$ & $0.414^{* *}$ & $0.249 * *$ & $0.751^{* *}$ & $0.486^{* *}$ & & \\
\hline \multirow[t]{4}{*}{ FM } & $2016 \mathrm{E} 1$ & $-0.237^{* *}$ & $-0.202^{* *}$ & $0.295^{* *}$ & $0.314^{* *}$ & $-0.320^{* *}$ & $-0.404^{* *}$ & -0.015 & 0.016 \\
\hline & 2015E2 & $-0.439 * *$ & $-0.334^{* *}$ & 0.088 & 0.049 & $-0.428^{* *}$ & $-0.390^{* *}$ & -0.102 & 0.047 \\
\hline & 2016E2 & $-0.443^{* *}$ & -0.124 & -0.053 & 0.093 & $-0.497^{* *}$ & $-0.261^{* *}$ & $-0.206^{* *}$ & 0.004 \\
\hline & $2016 \mathrm{E} 2^{*}$ & $-0.426^{* *}$ & $-0.277^{* *}$ & 0.063 & 0.142 & $-0.434^{* *}$ & $-0.482^{* *}$ & $-0.219^{* *}$ & -0.020 \\
\hline
\end{tabular}

$\overline{1 * \prime \prime},{ }^{* * \prime}$ indicate that the correlation is significant at 0.05 and 0.01 probability levels, respectively. RIL, recombinant inbred line population in maternal and paternal testcross trials, respectively; $B C$, backcross populations including maternal and paternal $B C(B C / M$ and $B C / P)$ populations. * : referred to $Q T L s$ identified in one $B C / M$ trial, the remaining QTLs identified in three BC/P trials 
with fiber uniformity (FU), fiber strength (FS) and fiber elongation (FE) in these populations except FU in 2016E1. However, fiber micronaire (FM) showed significant negative correlation with FL and FS in the populations. These results are similar to the previous researches (Liang et al. 2013; Shang et al. 2016d; Ma et al. 2019).

The correlations have similar tendency among $\mathrm{BC} / \mathrm{P}$ and $\mathrm{BC} / \mathrm{M}$ populations. In both $\mathrm{BC}$ populations, no significant correlation was detected between FU and FS. However, the majority of correlation values decreased in both $\mathrm{BC} / \mathrm{P}$ and $\mathrm{BC} / \mathrm{M}$ populations after backcrossing to either of parents.

\section{Single locus QTL analysis}

In four field trials, a total of 70 QTLs controlling fiber quality were detected in three corresponding populations of $\mathrm{BC} / \mathrm{P}$, RIL-P, BC/M and RIL-M, explaining 5.01\%$22.42 \%$ of phenotypic variance (PV) (Table S1, Fig. 1). These QTLs anchored on 17 chromosomes accordingly.

For fiber length, 9, 1 and 8 QTLs were identified in $\mathrm{BC} / \mathrm{P}, \mathrm{BC} / \mathrm{M}$ and RIL populations, respectively. The $q F L-C h r 5-2$ was simultaneously identified in 2015E2 and $2016 \mathrm{E} 1$ in the $\mathrm{BC} / \mathrm{P}$ population, explaining $7.46 \%$ and $7.82 \%$ of PV, respectively. The $q F L-C h r 1-2$ was simultaneously detected in 2016E1 and 2016E2 in RIL population, explaining $7.01 \%$ and $6.91 \%$ of PV, respectively. The $q F L-C h r 19-1$ was verified in two different populations, explaining $17.98 \%$ of $\mathrm{PV}$ in $\mathrm{BC} / \mathrm{P}$ population and $11.05 \%$ of PV in RIL population. Among eight QTLs detected in RIL population, three QTLs showed additive effects originated from GX1135 alleles whereas five QTLs showed additive effects offered by GX100-2 alleles. A total of three QTLs ( $q F L-C h r 5-1, q F L-C h r 5-2$ and $q F L-C h r 5-3)$ were distributed on chromosome 5 (Chr 5), and three QTLs ( $q F L-C h r 1-1, q F L-C h r 1-2$ and $q F L-C h r 1-3)$ were distributed on Chr 1.

A total of 14 QTLs were detected for fiber uniformity (FU) explaining $8.76 \%-11.86 \%$ of $\mathrm{PV}$, which distributed on 11 different chromosomes. Seven and five QTLs were identified in $\mathrm{BC} / \mathrm{P}$ and RIL populations, respectively. No common QTL was identified in multiple populations or multiple environments for FU. qFU-Chr6-1 increased FU providing alleles by GX1135 in RIL population, explaining $11.86 \%$ of PV.

For fiber strength (FS), a total of 10 QTLs were detected on seven chromosomes, explaining 6.27\%-22.09\% of PV. Two common QTLs were identified. The qFSChr3-1 was detected in BC/P population alone explaining high as $22.09 \%$ of PV in 2016E2. The qFS-Chr21-2 was detected in $\mathrm{BC} / \mathrm{P}, \mathrm{BC} / \mathrm{M}$ and RIL populations at the same time across 2016E1 and 2016E2, explaining 6.27\%$13.37 \%$ of PV.

In the $\mathrm{BC} / \mathrm{P}, \mathrm{BC} / \mathrm{M}$ and RIL populations, 8,3 and 8 QTLs were identified for fiber elongation (FE), respectively. Four common QTLs were detected at least in two populations, including $q F E-C h r 2-2, q F E-C h r 2-3$, $q F E-C h r 3-1$, and $q F E-C h r 21-2$. The $q F E-c h r 2-3$ was detected in $\mathrm{BC} / \mathrm{P}, \mathrm{BC} / \mathrm{M}$ and RIL populations and explained $7.02 \%$ of PV on average. The qFE-chr3-1 was also detected in $\mathrm{BC} / \mathrm{M}$ population in same environment of 2016E2, explaining $12.17 \%$ of $\mathrm{PV}$ in BC/P population. The qFE-Chr2-2 explained $22.42 \%$ of PV in RIL population in $2016 \mathrm{E} 1$ and was identified in $\mathrm{BC} / \mathrm{M}$ population in 2016E2. The $q F E-C h r 21-2$ was detected in $\mathrm{BC} / \mathrm{P}$ and RIL populations.

A total of 16 QTLs were detected for fiber micronaire. They were located on 12 different chromosomes. The qFM-Chr9-2 was identified in BC/M and RIL populations in 2016E2, explaining $21.91 \%$ of PV. At the same time, the QTLs were detected in three environments of 2015E2, 2016E1 and 2016E2. The QTL qFM-Chr15-1 was simultaneously detected in RIL population in 2016E1 and in BC/M population in 2016E2, explaining $12.36 \%$ and $5.46 \%$ of PV, respectively. All of six QTLs were detected in RIL population and fiber micronaire increased over 0.10 value, which donated increasing additive effect alleles by GX1135, containing $q F M-C h r 9-1$, qFM-Chr9-2, qFM-Chr12-1, qFM-Chr14-1, qFMChr14-2, and $q F M-C h r 15-1$. In summary, 35 QTL explained $5.01 \%-22.09 \%$ of $\mathrm{PV}$ on average in $\mathrm{BC} / \mathrm{P}$ population in 2015E2, 2016E1 and 2016E2. Among them, a total of 19 QTLs explained larger than $10.00 \%$ of PV. Then, we identified 10 QTLs in $\mathrm{BC} / \mathrm{M}$ population, explaining $5.31 \%-14.53 \%$ of PV. Thirty-five QTLs existed in RIL-P and RIL-M populations explaining $5.13 \%-21.91 \%$ of PV in four environments above. In total, 12 common QTLs were detected in multiple environments or in multiple populations of $B C / P, B C / M$, RIL-P or RIL-M populations including previous studies (Table S1, S5).

\section{Pleiotropic effects}

We also observed 5 pleiotropic regions controlling at least two fiber quality traits on $\mathrm{Chr}$ 9, Chr 18 and $\mathrm{Chr}$ 21 (Fig. 1). A pleiotropic region flanking with SWU15511- SWU15413 on Chr 9 increased the values for FL and FS, showing increased additive effects originated from alleles of GX100-2. The NAU2873CGR6771 on Chr 9 contributed alleles to FS but also increased the FM. The region of SWU0830-HAU2004CGR5602 contained $q F U$-Chr21-1 in BC/P population and qFE-Chr21-1 in RIL-P population. SWU0189CGR5808 on Chr 21 flanked along qFS-Chr21-1, qFSChr21-2 and qFE-Chr21-2, all of which showed increasing additive effects originated from alleles of GX100-2. The region of SWU15511- SWU15413 on Chr 18 controlled fiber length and fiber elongation at the same time. 


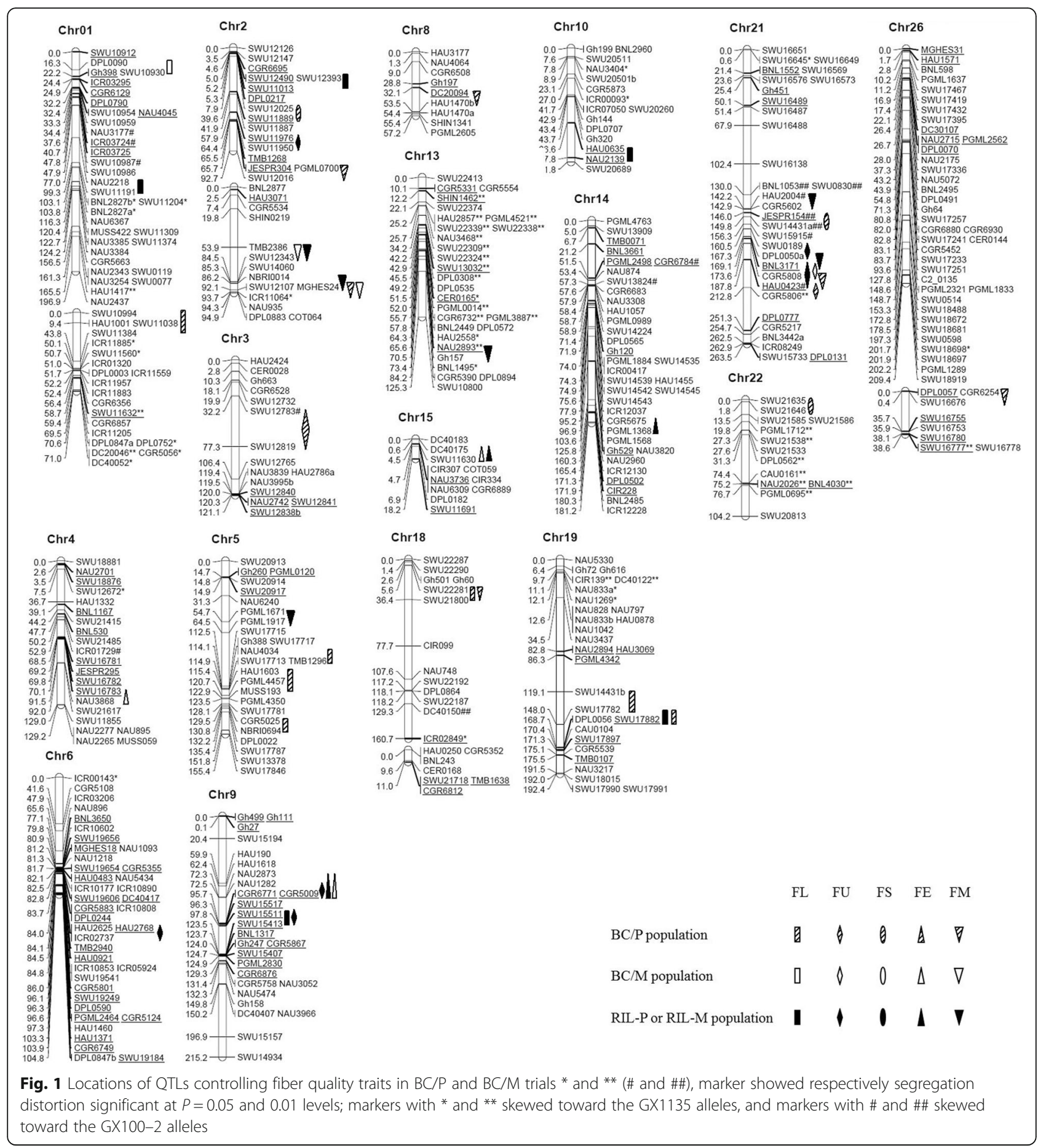

Digenic and environmental interaction in three $\mathrm{BC} / \mathrm{P}$ traits In three repetitive $\mathrm{BC} / \mathrm{P}$ trials, a total of 38 and $56 \mathrm{M}$ QTLs and environmental interactions (QTL $\times$ environment, $\mathrm{QE})$ were identified in $\mathrm{BC} / \mathrm{P}$ and RIL-P populations, respectively (Table 4, Table S2, Table S3). The result explained $2.53 \%-3.13 \%$ and $2.63 \%-3.41 \%$ of $\mathrm{PV}$, respectively, on average in the populations. Environmental effect prevailed in both $\mathrm{BC} / \mathrm{P}$ and RIL-P populations.
However, environment and M-QTL interacted with $1.24 \%$ of $\mathrm{PV}$ in the $\mathrm{BC} / \mathrm{P}$ population while with $0.90 \%$ of $\mathrm{PV}$ in RIL-P population.

Forty-six E-QTLs and 142 QQE (digenic interactions $\times$ environment) were respectively identified in $\mathrm{BC} / \mathrm{P}$ and RIL-P populations (Table S4, Table S5). Eighteen EQTLs and QQE explained $4.17 \%$ of PV on average in RIL-P population, while nine E-QTLs and QQE 
Table 4 Summary on M-QTL and E-QTLs controlling fiber quality traits in BC/P and RIL-P datasets in BC/P trials

\begin{tabular}{|c|c|c|c|c|c|c|}
\hline \multirow{2}{*}{$\begin{array}{l}\text { Trait } \\
\text { M-QTL }^{\text {a }}\end{array}$} & \multicolumn{3}{|c|}{$\mathrm{BC} / \mathrm{P}$} & \multicolumn{3}{|c|}{ RIL-P } \\
\hline & $\overline{n^{b}}$ & $V(A) \%^{c}$ & $\mathrm{~V}(\mathrm{AE}) / \%^{\mathrm{c}}$ & $\overline{n^{b}}$ & $V(A) \%^{c}$ & $V(A E) / \%^{c}$ \\
\hline$\overline{\mathrm{FL}}$ & 11 & 3.13 & 0.70 & 13 & 2.63 & 0.27 \\
\hline FU & 3 & 3.02 & 3.00 & 7 & 3.15 & 2.40 \\
\hline FS & 11 & 2.53 & 0.67 & 14 & 2.92 & 0.25 \\
\hline $\mathrm{FE}$ & 0 & - & - & 12 & 2.77 & 0.59 \\
\hline FM & 13 & 2.61 & 0.99 & 10 & 3.41 & 1.01 \\
\hline Mean & - & 2.82 & 1.34 & - & 2.98 & 0.90 \\
\hline E-QTL ${ }^{a}$ & $n^{b}$ & $\mathrm{~V}(\mathrm{AA}) / \%^{\mathrm{c}}$ & $\mathrm{V}(\mathrm{AAE}) \%^{\mathrm{c}}$ & $n$ & $V(A A) \%^{c}$ & $\mathrm{~V}(\mathrm{AAE}) / \%^{\mathrm{c}}$ \\
\hline $\mathrm{FL}$ & 16 & 3.44 & 0.30 & 52 & 4.33 & 0.22 \\
\hline FU & 11 & 1.93 & 2.12 & 0 & - & - \\
\hline FS & 5 & 3.32 & 0.52 & 44 & 4.15 & 0.24 \\
\hline $\mathrm{FE}$ & 1 & 0.76 & 0.01 & 18 & 4.42 & 0.24 \\
\hline FM & 13 & 3.44 & 0.73 & 28 & 3.77 & 0.23 \\
\hline Mean & - & 2.58 & 0.74 & - & 4.17 & 0.23 \\
\hline
\end{tabular}

${ }^{a}$ M-QTLs and E-QTLs refer to the main effect QTL and epistasis QTLs by environments. ${ }^{\mathrm{b}}$ The number of QTLs. ${ }^{\mathrm{C}} \mathrm{V}(\mathrm{A}) / \%, \mathrm{~V}(\mathrm{AAE}) / \%, \mathrm{~V}(\mathrm{AA}) / \%$ and $\mathrm{V}(\mathrm{AAE}) / \%$, the total proportion of phenotypic variation on average explained by single $\mathrm{QTL}$, epistatic QTLs (AA) and them by environments (AE or AAE) at the current scanning position, respectively

explained $2.58 \%$ of $\mathrm{PV}$ on average in $\mathrm{BC} / \mathrm{P}$ populations (Table 4). On average, the number of both types of M-QTL and E-QTL was larger in RIL populations than that in BC populations. The epistatic interactions contributed more to fiber quality than M-QTLs did in RIL-P population.

To sum up, 20 (42.5\%) and 101 (71.13\%) pairs of EQTLs and QQE contained M-QTLs and QEs in BC/P and RIL-P populations, respectively (Table 5). We detected about 3-fold epistatic QTLs in RIL populations than QTLs in BC/P population, and 19.01\% M-QTLs participated epistasis between M-QTL and M-QTL. Three types of epistasis were checked: I) both loci were M-QTLs; II) either locus between two loci was M-QTL; III) both loci were no M-QTLs (Shang et al. 2016d; Ma et al. 2019). Apparently, 27 (57.45\%) epistatic QTLs of

Table 5 Types of epistasis detected for fiber quality traits in the RIL-P and BC/P populations

\begin{tabular}{|c|c|c|c|c|c|c|c|c|}
\hline \multirow[t]{3}{*}{ Trait } & \multicolumn{8}{|c|}{ Type of epistasis $^{a}$} \\
\hline & \multicolumn{4}{|c|}{ RIL-P } & \multicolumn{4}{|c|}{$\mathrm{BC} / \mathrm{P}$} \\
\hline & I & $\|$ & III & $\overline{\text { Total }}$ & I & $\|$ & III & Total \\
\hline Fiber length & 8 & 29 & 15 & 52 & 2 & 5 & 9 & 16 \\
\hline Fiber uniformity & 0 & 0 & 0 & 0 & 2 & 5 & 4 & 11 \\
\hline Fiber strength & 10 & 22 & 12 & 44 & 0 & 1 & 5 & 6 \\
\hline Fiber elongation & 4 & 6 & 8 & 18 & 0 & 0 & 1 & 1 \\
\hline Micronaire & 5 & 17 & 6 & 28 & 1 & 4 & 8 & 13 \\
\hline Total & 27 & 74 & 31 & 142 & 5 & 15 & 27 & 47 \\
\hline
\end{tabular}

a type I, both loci were M-QTLs; type II, either locus among two loci was MQTL, and type III, both loci were no M-QTLs type III was the most popular type in epistatic styles in $\mathrm{BC} / \mathrm{P}$ population whereas it was $31(52.11 \%)$ epistatic QTLs of type II in RIL-P population (Table 5). The results indicated that epistasis played more vital role in improving fiber quality in RIL populations of upland cotton. The result was consistent to the previous result that epistatic QTLs with significant additive $\times$ additive effects were identified for fiber quality traits (Wang et al. 2017; Ma et al. 2019).

\section{Discussion}

In present study, the paternal $\mathrm{BC}(\mathrm{BC} / \mathrm{P})$ population was constructed to explore the genetic mechanism of fiber quality, following previous studies in maternal $\mathrm{BC}(\mathrm{BC} /$ M) population (Shang et al. 2016d; Ma et al. 2017, 2019). The backcross design has the obvious advantages: (I) dissecting the genetic components between paternal and maternal backcross populations; (II) identifying more novel QTLs for important traits using multiple corresponding populations (BC/P, BC/M and RIL) originated from the same hybrid; and (III) allowing to generate enough hybrid seeds when needed, similar to $\mathrm{IF}_{2}$ population. Here we detected 19 and 8 QTLs alone in $\mathrm{BC} / \mathrm{P}$ and $\mathrm{BC} / \mathrm{M}$ populations, respectively. Three QTLs shared in both $\mathrm{BC}$ populations for fiber strength and fiber elongation, including qFS-Chr21-2, qFE-Chr2-3 and qFE-Chr3-1. The result indicated that the remaining elite alleles (84.21\%) showed increasing additive effects originated from male parent for fiber quality in $\mathrm{BC} / \mathrm{P}$ population. Therefore, present study was significant in separating novel elite alleles of male parent for fiber quality.

The identification of stable QTLs (including common QTLs) across multiple environments and multiple populations plays an essential role in marker-assisted selection (MAS) (Jamshed et al. 2016). In present study, a total of 12 common QTLs were simultaneously identified in more than one environment(s) or population(s) (Table S1). They distributed on Chr 1, Chr 2, Chr 3, Chr 5, Chr 9, Chr 15, Chr 19 and Chr 21. A total of 13 single locus QTLs (35.14\%) for fiber quality were common in comparison with the previous studies in multiple years and multiple locations shown in Table S6 (Shang et al. 2016d; Ma et al. 2017). The QTLs verified each other in the RIL population and its $\mathrm{BC}$ progenies, suggesting that it is reasonable and effective to map QTLs using different populations across multiple environments and multiple years. The experiment design and the continuous study in our lab verified these results in present study. Among the 13 QTLs, 10 QTLs explained the larger than $10 \%$ of PV. Five QTLs were identified in Shang et al.' results (2016d) and Ma et al.' results (2017), including qFL-Chr5-1, qFL-Chr5-2, qFL-Chr5-3, qFS-Chr21-1 and qFE-Chr2-1 (Table S6). The QTL increased 0.31 
$\mathrm{mm}$ fiber length (FL) on average, suggesting the significant roles and important regions for FL. In addition, $q L P$ Chr5-3 and qBNP-Chr5-2 increased the lint percentage and the boll number per plant, along $333 \mathrm{~kb}$ (48 genes) pleiotropic regions along the SSR marker TMB1296 (Ma et al. 2019). However, no gene was reported along the region. These suggested QTLs were valuable for follow-up breeding program, so as to facilitate fine mapping and favorable gene pyramiding project (Shao et al. 2014).

In addition, the marker BNL1495 flanking qFE-Chr13-1 in present study was the same to the marker flanking $q F L$ chr13-2 in Liang et al.' study (2013). The marker NAU3384 of $q F S$-Chr1-1 flanked to the NAU3385, which was detected for lint percentage and lint index in Chr 1 in the previous study (Zhang et al. 2013). Nine SSR markers flanking QTLs for fiber quality traits in Tang's work were in common with our genetic map (2015). The markers PGML3120 and PGML4657 flanking qFS24.1, qFE24.1 and qFM24.1, were linked to the SWU13256 and SWU13267 of qFL-Chr24-1 and qFE-Chr24-1 in present study. The stable $q F S-C h r 21-2$ in present study shared a common SSR marker BNL3171 flanking a stable QTL of $q U H M-21-1$ controlling fiber length in previous study (Wang et al. 2017). The $q F S-C h r 21-2$ was also detected in 2015E3 for improving fiber strength (Ma et al. 2017). The qFS07.1 controlling fiber strength was fine-mapped to a 62.6-kb region, but no same marker was found with NAU3181 and SHIN0376 for $q F U$-Chr7-1 in the present study (Fang et al. 2017c). These SSR markers are valuable for fiber quality breeding in terms of previous studies in different varieties of cotton. The classic method for mapping QTL and tagging genes had improved plant improvement programs by using marker-assisted selection (MAS). Indeed, in many previous studies involving SSR markers and/or phenotypes, QTLs were obtained for important traits (Zhang et al. 2005; Zhang et al. 2015c; Xu et al. 2017). The artificial selected plants had been bred for varieties using MAS to major crop breeding programs even by long selection cycles. With the decreasing cost and increasing SNP density by nextgeneration sequencing approach, the strategy of genomic selection (GS) will consider genetic effects across whole genome. Much improvment in accuracy and efficiency could be expected if combined the results from both the classic method and GS strategy.

In present study, a total of 35 QTLs were detected in the $\mathrm{BC} / \mathrm{P}$ population. Taking all the detected QTLs together, 23 (65.71\%) novel QTLs were identified from $\mathrm{BC} / \mathrm{P}$ populations alone, and $9(25.71 \%)$ were detected in both $\mathrm{BC} / \mathrm{M}$ and $\mathrm{BC} / \mathrm{P}$ populations. The results indicated that many unique QTLs can be detected solely by backcrossing male parents to $F_{1}$ plants, which are available for marker-assistant breeding. Interestingly, 5 in 10 $(50 \%)$ and 28 in $35(80 \%)$ QTLs were only detected in $\mathrm{BC} / \mathrm{M}$ and RIL populations, respectively.
The epistatic effects and environmental interactions existed simultaneously for fiber quality traits as well as other traits. At two-locus level, we detected a number of interactions under environments for fiber quality traits in both populations (Table 4). Three types of epistasis combinations were observed (Table 5). However, epistasis QTLs influenced fiber quality by Type III (57.45\%) in BC/P population. Differently, epistasis influenced fiber quality by Type I and Type II (71.13\%) in RIL population. In addition, 3fold epistasis QTLs was detected in RIL-P population. The results indicate that epistasis played roles in different genetic modes to control fiber quality. In particular, no E-QTL was identified for fiber uniformity (FU). Another interesting result is that epistasis is another vital genetic effect affecting fiber quality traits (Shang et al. 2016d). Similar to previous study, Wang et al. (2006) indicated that both epistasis effect and single-locus effect of QTLs played an important genetic role in cotton fiber quality.

In present study, five QTLs increased fiber micronaire (FM) values from 0.06 to 0.18 (Table S1). Mean values ranged 4.71-5.18 on average in the RIL-P and $\mathrm{BC} / \mathrm{P}$ populations of Xinza 1. In other words, fiber quality ranks from B grade (3.5-3.6, 4.3-4.9) to $C$ grade $(<3.4$, $>5.0)$ for fiber micronaire. At the same time, FM displayed negative correlation with FL, FU, FS and FE. Therefore, we should avoid exploiting the QTL regions in breeding project. Larger lint yield potential and well fiber quality are the key aims in cotton breeding program, and negative correlation between yield and fiber quality hinders genetic gains in cotton breeding (Yang et al. 2015). Many important heterotic loci were detected in our previous studies (Shang et al. 2016a; Ma et al. 2019), and some heterotic loci were also identified accounting for improving fiber quality (Shang et al. 2016d; $\mathrm{Ma}$ et al. 2017). The pleiotropic regions should be paying more attention in further research so as to improve fiber quality and to increase yield in breeding program.

\section{Conclusions}

In present study, the paternal $\mathrm{BC}(\mathrm{BC} / \mathrm{P})$ population was constructed to explore the genetic mechanism of fiber quality, and was detected 19 and 8 QTLs alone in BC/P and $\mathrm{BC} / \mathrm{M}$ populations, respectively. Three QTLs shared in both $\mathrm{BC}$ populations for fiber strength and fiber elongation, including qFS-Chr21-2, qFE-Chr2-3 and $q F E-C h r 3-1$. The present study was significant in separating novel elite alleles of male parent for fiber quality.

\section{Supplementary information}

Supplementary information accompanies this paper at https://doi.org/10. 1186/s42397-020-00060-6.

Additional file 1: Table S1. Single-locus QTLs in paternal and maternal backcross experiments by composite interval mapping method. 
Additional file 2: Table S2. Main effects and environmental interactions detected for fiber quality traits in RIL-P population.

Additional file 3: Table S3. Epitasis effects and environmental interactions detected for fiber quality traits in RIL-P population.

Additional file 4: Table S4. Main effects and environmenta interactions detected for fiber quality traits in BC/P population.

Additional file 5: Table S5. Epitasis effects and environmental interactions detected for yield and its components in BC/P population.

Additional file 6: Table S6. Common single locus QTLs in comparison with the previous studies in multiple years and locations.

Additional file 7: Table S7. Phenotype values of fiber quality traits of BC/P population in three field trials in this study.

Additional file 8: Table S8. Phenotype values of fiber quality traits in field trial of BC/M population in this study.

Additional file 9: Table S9. Genotypes of RIL (A), BC/M (B) and BC/P (C) populations.

\section{Abbreviations}

BC: Backcross; BC/P: Paternal backcross population; BC/M: Maternal backcross population; Chr: Chromosome; CIM: Composite interval mapping; E-

QTLs: Epistatic QTLs; FE: Fiber elongation; FL: Fiber length; FM: Micronaire; FS: Fiber strength; FU: Fiber uniformity; GS: Genomic selection; LOD: Logarithm of odds; M-QTL: Main-effect QTL; MAS: Marker-assisted selection; PV: Phenotype variation; QE: QTL $\times$ environmental interaction; QTL: Quantitative trait loci; QQEs: Epistatic QTLs $\times$ environments; RIL: Recombinant inbred line

\section{Acknowledgements}

We thank Cai Shihu (China Agricultural University), Xu Dongyong and Lu Huaiyu (Guoxin Seed Company Ltd., Cangzhou, Hebei province) for their contributions on field experiments and data acquisition. Thanks to Liang Qingzhi, Shang Liangguang, Abduweli Abdugheni, Wang Xiaocui (China Agricultural University) for the contributions in SSR marker evaluation and map construction, Wang Kunbo and Liu Fang (Institute of Cotton Research, Chinese Academy of Agricultural Sciences) for providing part of SSR markers, Dr. Zhang Zhengsheng (Southwest University) for providing SWU and ICR SSR primers.

\section{Authors' contributions}

Hua JP designed the experiment. Ma LL performed the experiments, analyzed the data and prepared the manuscript. Su Y, Nie HS, Cui YP, Cheng $C$ and ljaz B attended field experiments and data collection. Hua JP provided experimental platform and revised the manuscript. All authors read and approved the final manuscript

\section{Funding}

This research was supported by the National Key R \& D Program for Crop Breeding (2016YFD0101407) to Hua JP.

\section{Availability of data and materials}

All data generated or analyzed in this study included in published article and additional files. All of our raw data are available as Supporting Information Table S7 and Table S8 for phenotypes used in multiple populations and Supporting Information Table S9 for genotypes for RIL, BC/M and BC/P population (Shang et al. 2016a; Ma et al. 2019).

\section{Ethics approval and consent to participate}

Not applicable.

\section{Consent for publication}

Not applicable.

\section{Competing interests}

The authors declare that they have no competing interests.
Received: 21 December 2019 Accepted: 22 June 2020

Published online: 25 August 2020

\section{References}

Ali I, Teng Z, Bai Y, et al. A high density SLAF-SNP genetic map and QTL detection for fibre quality traits in Gossypium hirsutum. BMC Genomics. 2018; 19:879. https://doi.org/10.1186/s12864-018-5294-5.

Du X, Huang G, He S, et al. Resequencing of 243 diploid cotton accessions based on an updated a genome identifies the genetic basis of key agronomic traits. Nat Genet. 2018;50:796-802. https://doi.org/10.1038/s41588-018-0116-x.

Fang $\mathrm{L}$, Gong $\mathrm{H}, \mathrm{Hu} Y$, et al. Genomic insights into divergence and dual domestication of cultivated allotetraploid cottons. Genome Biol. 2017a;18:33. https://doi.org/10.1186/s13059-017-1167-5.

Fang L, Wang Q, Hu Y, et al. Genomic analyses in cotton identify signatures of selection and loci associated with fiber quality and yield traits. Nat Genet. 2017b;49:1089-98. https://doi.org/10.1038/ng.3887.

Fang X, Liu X, Wang X, et al. Fine-mapping gFS07. 1 controlling fiber strength in upland cotton (Gossypium hirsutum L.). Theor Appl Genet. 2017c;130:795-806. https://doi.org/10.1007/s00122-017-2852-1.

Hu Y, Chen J, Fang L, et al. Gossypium barbadense and Gossypium hirsutum genomes provide insights into the origin and evolution of allotetraploid cotton. Nature Genet. 2019:51:739-48. https://doi.org/10.1038/s41588-019-0371-5.

Hua JP, Xing YZ, Wu WR, et al. Single-locus heterotic effects and dominance by dominance interactions can adequately explain the genetic basis of heterosis in an elite rice hybrid. Proc Natl Acad Sci U S A. 2003;100:2574-9. https://doi. org/10.1073/pnas.0437907100

Hua JP, Xing YZ, Xu CG, et al. Genetic dissection of an elite rice hybrid revealed that heterozygotes are not always advantageous for performance. Genetics. 2002;162:1885-95.

ljaz B, Zhao N, Kong J, Hua J. Fiber quality improvement in upland cotton (Gossypium hirsutum L.): quantitative trait loci mapping and marker assisted selection application. Front Plant Sci. 2019;10:1585. https://doi.org/10.3389/ fpls.2019.01585.

Jamshed MJ, Jia F, Gong JW, et al. Identification of stable quantitative trait loci (QTLs) for fiber quality traits across multiple environments in Gossypium hirsutum recombinant inbred line population. BMC Genomics. 2016;17:197. https://doi.org/10.1186/s12864-016-2560-2.

Lacape JM, Nguyen TB, Courtois B, et al. QTL analysis of cotton fiber quality using multiple Gossypium hirsutum $\times$ Gossypium barbadense testcross generations. Crop Sci. 2005;45:123-40. https://doi.org/10.2135/cropsci2005.0123a.

Li C, Dong YT, Zhao TL, et al. Genome-wide SNP linkage mapping and QTL analysis for fiber quality and yield traits in the upland cotton recombinant inbred lines population. Front Plant Sci. 2016;7:1356. https://doi.org/10.3389/ fpls.2016.01356.

Li FG, Fan GY, Lu CR, et al. Genome sequence of cultivated upland cotton (Gossypium hirsutum TM-1) provides insights into genome evolution. Nat Biotechnol. 2015;33:524-30. https://doi.org/10.1038/nbt.3208.

Li FG, Fan GY, Wang KB, et al. Genome sequence of the cultivated cotton Gossypium arboreum. Nat Genet. 2014;46:567-72. https://doi.org/10.1038/ng.2987.

Liang QZ, Hu C, Hua H, et al. Construction of a linkage map and QTL mapping for fiber quality traits in upland cotton (Gossypium hirsutum L.). Chinese Sci Bull. 2013;58:3233-43. https://doi.org/10.1007/s11434-013-5807-1.

Liang QZ, Shang LG, Wang YM, Hua JP. Partial dominance, overdominance and epistasis as the genetic basis of heterosis in upland cotton (Gossypium hirsutum L.). PLoS One. 2015;10:e0143548. https://doi.org/10.1371/journal. pone.0143548.

Liu X, Zhao B, Zheng HJ, et al. Gossypium barbadense genome sequence provides insight into the evolution of extra-long staple fiber and specialized metabolites. Sci Rep. 2015:5:14139. https://doi.org/10.1038/srep14139.

Ma LL, ljaz B, Wang YM, Hua JP. Dynamic QTL analysis and validation for plant height using maternal and paternal backcrossing populations in upland cotton. Euphytica. 2018a;214:167. https://doi.org/10.1007/s10681-018-2243-y.

Ma LL, Wang YM, ljaz B, Hua JP. Cumulative and different genetic effects contributed to yield heterosis using maternal and paternal backcross populations in upland cotton. Sci Rep. 2019;9:3984. https://doi.org/10.1038/s41598-019-40611-9.

Ma LL, Zhao YP, Wang YM, et al. QTLs analysis and validation for fiber quality traits using maternal backcross population in upland cotton. Front Plant Sci. 2017:8:2168. https://doi.org/10.3389/fpls.2017.02168

Ma Z, He S, Wang X, et al. Resequencing a core collection of upland cotton identifies genomic variation and loci influencing fiber quality and yield. Nat Genet. 2018b;50:803-13. https://doi.org/10.1038/s41588-018-0119-7. 
Mei HW, Li ZK, Shu QY, et al. Gene actions of QTLs affecting several agronomic traits resolved in a recombinant inbred rice population and two backcross populations. Theor Appl Genet. 2005;110:649-59. https://doi.org/10.1007/s00122-004-1890-7.

Ning ZY, Chen H, Mei HX, Zhang TZ. Molecular tagging of QTLs for fiber quality and yield in the upland cotton cultivar Acala-Prema. Euphytica. 2014;195: 143-56. https://doi.org/10.1007/s10681-013-0990-3.

Paterson AH, Wendel JF, Gundlach H, et al. Repeated polyploidization of Gossypium genomes and the evolution of spinnable cotton fibres. Nature. 2012;492:423-7. https://doi.org/10.1038/nature11798.

Said JI, Knapka JA, Song MZ, Zhang JF. Cotton QTLdb: a cotton QTL database for QTL analysis, visualization, and comparison between Gossypium hirsutum and G. hirsutum × G. barbadense populations. Mol Genet Genom. 2015;290:161525. https://doi.org/10.1007/s00438-015-1021-y.

Said Jl, Lin ZX, Zhang XL, et al. A comprehensive meta QTL analysis for fiber quality, yield, yield related and morphological traits, drought tolerance, and disease resistance in tetraploid cotton. BMC Genomics. 2013;14:776. https://doi.org/10.1186/1471-2164-14-776.

Shahzad K, Li X, Qi T, et al. Genetic analysis of yield and fiber quality traits in upland cotton (Gossypium hirsutum L.) cultivated in different ecological regions of China. J Cotton Res. 2019;2:14. https://doi.org/10.1186/s42397-019-0031-4.

Shang LG, Liang QZ, Wang YM, et al. Identification of stable QTLs controlling fiber traits properties in multi-environment using recombinant inbred lines in Upland cotton (Gossypium hirsutum L.). Euphytica. 2015;205:877-88. https:// doi.org/10.1007/s10681-015-1434-z.

Shang LG, Liang QZ, Wang YM, et al. Epistasis together with partial dominance, over-dominance and QTL by environment interactions contribute to yield heterosis in upland cotton. Theor Appl Genet. 2016a;129:1429-46. https://doi. org/10.1007/s00122-016-2714-2.

Shang LG, Wang YM, Cai SH, et al. Genetic analysis of upland cotton dynamic heterosis for boll number per plant at multiple developmental stages. Sci Rep. 2016b;6:35515. https://doi.org/10.1038/srep35515.

Shang LG, Wang YM, Cai SH, et al. Partial dominance, overdominance, epistasis and QTL by environment interactions contribute to the heterosis in two upland cotton hybrids. G3: Genes, Genomes, Genetics. 2016c;6:499-507. https://doi.org/10.1534/g3.115.025809

Shang LG, Wang YM, Wang XC, et al. Genetic analysis and stable QTL detection on fiber quality traits using two recombinant inbred line populations and their backcross progeny in upland cotton. G3: Genes, Genomes, Genetics. 2016d;6:2717-24. https://doi.org/10.1534/g3.116.031302 .

Shao QS, Zhang FJ, Tang SY, et al. Identifying QTL for fiber quality traits with three upland cotton (Gossypium hirsutum L.) populations. Euphytica. 2014; 198:43-58. https://doi.org/10.1007/s10681-014-1082-8.

Shi Y, Liu A, Li J, et al. Examining two sets of introgression lines across multiple environments reveals background-independent and stably expressed quantitative trait loci of fiber quality in cotton. Theor Appl Genet. 2020;133: 2075-93. https://doi.org/10.1007/s00122-020-03578-0

Su JJ, Li LB, Pang CY, et al. Two genomic regions associated with fiber quality traits in Chinese upland cotton under apparent breeding selection. Sci Rep. 2016;6:38496. https://doi.org/10.1038/srep38496.

Sun FD, Zhang JH, Wang SF, et al. QTL mapping for fiber quality traits across multiple generations and environments in upland cotton. Mol Breeding. 2012;30:569-82. https://doi.org/10.1007/s11032-011-9645-z .

Tan ZY, Fang XM, Tang SY, et al. Genetic map and QTL controlling fiber quality traits in upland cotton (Gossypium hirsutum L.). Euphytica. 2015;203:615-28. https://doi.org/10.1007/s10681-014-1288-9.

Tang SY, Teng ZH, Zhai TF, et al. Construction of genetic map and QTL analysis of fiber quality traits for upland cotton (Gossypium hirsutum L.). Euphytica. 2015;201:195-213. https://doi.org/10.1007/s10681-014-1189-y.

Wang B, Guo W, Zhu X, et al. QTL mapping of fiber quality in an elite hybrid derived-RIL population of upland cotton. Euphytica. 2006;152(3): 367-378. https://doi.org/10.1007/s10681-006-9224-2.

Wang BH, Draye X, Zhuang ZM, et al. QTL analysis of cotton fiber length in advanced backcross populations derived from a cross between Gossypium hirsutum and G mustelinum. Theor Appl Genet. 2017;130:1297-308. https:// doi.org/10.1007/s00122-017-2889-1.

Wang HT, Huang C, Zhao WX, et al. Identification of QTL for fiber quality and yield trait s using two immortalized backcross populations in upland cotton. PLoS One. 2016;11:e0166970. https://doi.org/10.1371/journal.pone.0166970.

Wang KB, Wang ZW, Li FG, et al. The draft genome of a diploid cotton Gossypium raimondii. Nat Genet. 2012a;44:1098-103. https://doi.org/10.1038/ng.2371.
Wang M, Tu L, Yuan D, et al. Reference genome sequences of two cultivated allotetraploid cottons, Gossypium hirsutum and Gossypium barbadense. Nat Genet. 2019;51:224-9. https://doi.org/10.1038/s41588-018-0282-x.

Wang S, Basten CJ, Zeng ZB. Windows QTL Cartographer V2.5-011. Raleigh, NC: Department of Statistics, North Carolina State University; 2012b. http:// statgen.ncsu.edu/qtlcart/WQTLCart.htm.

Wu JX, Gutierrez OA, Jenkins JN, et al. Quantitative analysis and QTL mapping for agronomic and fiber traits in an Rl population of upland cotton. Euphytica. 2009;165:231-45. https://doi.org/10.1007/s10681-008-9748-8.

Xu P, Gao J, Cao ZB, et al. Fine mapping and candidate gene analysis of qFL-chr1, a fiber length QTL in cotton. Theor Appl Genet. 2017;130:1309-19. https:// doi.org/10.1007/s00122-017-2890-8.

Yang XL, Zhou XD, Wang XF, et al. Mapping QTL for cotton fiber quality traits using simple sequence repeat markers, conserved intron-scanning primers, and transcript-derived fragments. Euphytica. 2015;201:215-30. https://doi.org/ 10.1007/s10681-014-1194-1.

Yu J, Jung S, Cheng CH, et al. CottonGen: a genomics, genetics and breeding database for cotton research. Nucleic Acids Res. 2014;42:D1229-36. https:// doi.org/10.1093/nar/gkt1064.

Yuan DJ, Tang ZH, Wang MJ, et al. The genome sequence of Sea-Island cotton (Gossypium barbadense) provides insights into the allopolyploidization and development of superior spinnable fibres. Sci Rep. 2016;5:17662. https://doi. org/10.1038/srep17662.

Zeng ZB. Precision mapping of quantitative trait loci. Genetics. 1994;136:1457-68.

Zhang JF, Yu JW, Pei WF, et al. Genetic analysis of Verticillium wilt resistance in a testcross inbred line population and a meta-analysis of quantitative trait loci for disease resistance in cotton. BMC Genomics. 2015c;16:577. https://doi.org/ 10.1186/s12864-015-1682-2.

Zhang T, Qian N, Zhu X, et al. Variations and transmission of QTL alleles for yield and fiber qualities in upland cotton cultivars developed in China. PLoS One. 2013;8:e57220. https://doi.org/10.1371/journal.pone.0057220.

Zhang TZ, Hu Y, Jiang WK, et al. Sequencing of allotetraploid cotton (Gossypium hirsutum L. acc. TM-1) provides a resource for fiber improvement. Nat Biotechnol. 2015a;33:531-7. https://doi.org/10.1038/nbt.3207.

Zhang Z, Li JW, Muhammad J, et al. High resolution consensus mapping of quantitative trait loci for fiber strength, length and micronaire on chromosome 25 of the upland cotton (Gossypium hirsutum L.). PLoS One. 2015b;10:e0135430. https://doi.org/10.1371/journal.pone.0135430.

Zhang ZS, Xiao YH, Luo M, et al. Construction of a genetic linkage map and QTL analysis of fiber-related traits in upland cotton (Gossypium hirsutum L.). Euphytica. 2005;144:91-9. https://doi.org/10.1007/s10681-005-4629-х.

Ready to submit your research? Choose BMC and benefit from:

- fast, convenient online submission

- thorough peer review by experienced researchers in your field

- rapid publication on acceptance

- support for research data, including large and complex data types

- gold Open Access which fosters wider collaboration and increased citations

- maximum visibility for your research: over $100 \mathrm{M}$ website views per year

At $\mathrm{BMC}$, research is always in progress.

Learn more biomedcentral.com/submissions 